\title{
Intelligent and Adaptive Tutoring Systems: How to Integrate Learners
}

\author{
Mehri Mohammad Bagheri ${ }^{1, *}$ \\ ${ }^{1}$ Faculty of Foreign Languages and Culture, Philipps University of Marburg, Marburg, \\ Germany \\ *Correspondence: Faculty of Foreign Languages and Culture, Philipps University of Marburg, \\ Marburg, Germany. E-mail: mmbaqeri@gmail.com
}

Received: February 10, 2015 Accepted: March 24, 2015 Published: April 10, 2015

doi:10.5296/ije.v7i2.7079 URL: http://dx.doi.org/10.5296/ije.v7i2.7079

\begin{abstract}
This article is a non-expert overview of intelligent technology-based instructional machines, from the first stages of their emergence up to this date. It is attempted to demonstrate their trend of evolution, and how the advances in other related fields such as education, psychology, and computer science affected them and contributed to their progress. It starts by an account of the concept of intelligent technology-based instruction and continues by defining the notion of learner integration in such systems. Furthermore, Intelligent Tutoring Systems (ITSs) are pictured: how they evolved, their architecture, and how they contributed to the field of Adaptive Learning Systems (ALSs). Finally, a number of adaptive learning platforms which are the state of the art and currently used by large numbers of actual users are introduced and described.
\end{abstract}

Keywords: intelligent technology-based instruction, learner integration, intelligent tutoring systems, adaptive learning systems 


\section{Introduction}

Nowadays, the need to education is unlike any period before because the increasing advances in technology and science demand people who continuously learn and update their knowledge repertoire. Learning is not, similar to the past, limited to school days, and people need to have access to new or previously learned information at any time and place. Less than fifty years ago, such unlimited access to information seemed like a fancy and untouchable dream because education and learning were tied to knowledgeable people and instructional materials, and such people or materials were only accessible in specific places and times.

The emergence of computer and internet technologies; however, realized this dream. The first and most important thing they did was making information accessible to anyone who had access to them. Internet, as it is today, emerged around 1980's and it quickly spread around the globe. It enabled its users to have access to others' information, contribute to it with their own information, and be in contact with other users. In parallel, the advances in computer technology brought about cheap and high-speed personal computers and mobiles and soon, many schools, offices, and homes were equipped with them. They enabled people to access this large pool of information from any place and in any time.

Apart from technology progress, research in the field of human learning widened the knowledge of educationists and psychologists beyond the Behaviorist views of learning, and learners were confirmed to be more than passive receivers of information and respondents to environmental stimuli. In fact, the Cognitivist and Constructivist theories of learning showed that learners have the capacity of processing the information and building their individual knowledge bases, and they verified the importance of tailoring instruction to the needs of individual learners rather than prescribing a one-size-fits-all approach.

Additionally, the pioneering research by Bloom demonstrated the superiority of one-to-one over group instruction with outstanding results (Bloom, 1984). Bloom conducted experiments with different groups of learners in different learning situations and concluded that the ideal form of instruction is when an instructor instructs a person-or two to three people-rather than a group of people. The reason is that good instruction is not only a matter of conveying information, but also adapting the instruction to individual learners. A reality that is better realizable in one-to-one learning conditions. However, one-to-one tutoring has always been hard to realize in real-world educational setting due to the large difference between the number of instructors and learners and the high costs of providing one instructor for each learner.

Such scientific findings and technology advances, coupled with the emergence of Artificial Intelligence (AI), triggered the idea of designing technology-based instructional machines which are intelligent enough to adapt their instruction to learners in order to provide the benefits of one-to-one tutoring. The use of machines and computers for instructional purposes was already a mainstream at the time of AI emergence, but was limited to static and inflexible systems which were not intelligent and adaptive. After AI came on the stage, numerous systems with different purposes, theoretical backgrounds, architecture, and under different titles were designed and developed, and while some did not acquire much success, others have been quite successful in some domains. 


\section{Review of the Field}

\subsection{Intelligent Technology-based Instruction}

The term "intelligent" when applied to instructional machines refers to any behavior which if performed by a human instructor would be considered as "good teaching" (Elsom-Cook, 1987). The idea to create intelligent instructional machines dates back to the early days of computer emergence and it strengthened with the advent of AI field. From the first attempts to the present time, intelligence has been realized in instructional machines in different degrees and forms.

In early mechanical teaching machines, intelligence was only a matter of splitting a bigger task into smaller sub-tasks and presenting the tasks to the learners in a fixed order until they could complete the whole task. In these early systems, no feedback was provided, or if provided, it was not intelligently generated and was only a result of what was pre-defined and programmed in the system (Nwana, 1990).

True intelligence in instructional machines appeared much later, as AI came into existences. During the 1970s and 80s, AI researchers made great strides in the domain of intelligent technology-based instruction, and created systems under the title of intelligent tutoring systems (ITSs) with an almost fixed architecture that were intelligent with regard to their subject matter (What they teach), learners (Who they teach), and instructional methodologies (How they teach it) (Self, 1974). Many different ITSs were designed and developed in AI labs during that period, showing outstanding degrees of intelligence; however, very few of them could find a place out of AI labs. One reason for their lack of practicality was that they were severely dominated by AI and computer science fields without enough cooperation with other related fields. Researchers began to realize that intelligent technology-based instruction is a multidisciplinary rather than a single-disciplined field and it requires the insights from psychology and education, as well as AI and computer science.

Adaptive learning systems (ALSs) are a new generation of ITSs and a hot topic in intelligent technology-based instruction which are intended to compensate for the inadequacies of ITSs. ALSs are usually concept instructors rather than problem solving tutors and are designed to adapt their content and teaching to the learning styles and preferences, as well as knowledge, of learners. Their architecture is similar to ITSs, with the addition of adaptive hypermedia technologies which enable them to give learners personalized access to World Wide Web information.

\subsection{How to Integrate Learners}

Learner integration can happen at different layers of an intelligent technology-based instructional machine and with different levels of sophistication. By the term "learner integration" the author of this article means any consideration of the learner by the instructional machine in order to make the content and delivery of information more adapted and adjusted to the needs and preferences of individual learners.

The simplest form of learner integration is providing options that enable learners to alter the 
interface appearance of the system such as the background color, font color, font size, presence or not presence of voice; to mention a few. The aim of such adaptations is to make the learning environment more pleasant for individual tastes. At a more sophisticated level, integration or adaptation can occur by giving the learners a choice of the materials which they see as best for their learning goals. This type of integration has been realized in instructional machines in different forms and levels and is currently a main part of ALSs.

Learner integration is also applicable without the learners paying attention and as they interact with the system. In this situation, the system actively and silently monitors the learners as they do a task or solve a problem, and keeps a history of their activities and what they have or have not learned. This history of activities is stored in the Student Model (may have different names in different systems) of these systems and becomes a basis for giving the learners immediate feedback or determining the level of difficulty of the task. This type of learner integration; similarly, has devoted a vast field of research to itself and has been realized in different degrees and forms in different systems.

Yet, an equally or even more important form of learner integration which has been largely ignored until recent years is to provide a model of the learners to adapt the content and content delivery of the system to the needs and learning styles of the learners. Previous systems had such a learner model or student model, but the information in it mostly included information about what the learners know or do not know, and what they have learnt or have not learnt. This; however, is not enough because a good human tutor, beside such information, knows how to teach different learners while considering their personal differences and learning styles. Also, the previous systems, though giving their learners some levels of freedom in choosing their content, were at the same time very restrictive in this regard and the content, if there was any content at all, was mostly limited to what existed in their Domain Model. Current systems, using the technology of adaptive hypermedia, have given students access to more information available on World Wide Web.

This last type of integration has recently been emphasized in ALSs which are currently the pinnacle of technology-based instruction field. These systems still follow the familiar architecture of ITSs, but their emphasis has shifted from the system itself to the learner. In other words, instead of looking at the learners as passive receivers of information and directed by the system, they attempt to give them more freedom in choice of material and learning goals based on their individual preferences.

\subsection{Intelligent Tutoring Systems}

ITSs are instructional machines designed based on the principles of AI, education, and psychology fields. These systems perform instructional acts and decisions which if performed by human tutors would be considered as intelligent or good instruction (Elsom-Cook, 1987 \& Nwana, 1990). Other definitions include Psotka et al. (1988) who defined ITSs as instructional systems which are designed to give the learner immediate instruction and feedback, almost without any human interference.

These systems are also labeled by some researchers as ICAI (Intelligent Computer-Assisted 


\section{Macrothink}

Instruction) which has revolved from the more traditional CAI field (Urban-Lurain, 1996 [INT 19]). However, the term ITS was coined by Sleeman and Brown (1982) and gained more support than ICAI after being confirmed in the International Conference on Intelligent Tutoring Systems held in 1988 in Montreal, Canada (Nwana, 1990) and the same term is used throughout this article to refer to such systems.

ITSs have seen different architectural structures since their emergence and almost every ITS is different from the rest in some details, but a structure which exists more or less in most ITSs is the one consisted of Domain, Student, Tutor, and User Interface models. In short, a domain model is part of ITS which holds information about the subject matter which is taught to the learners; a student model has information about individual learners and updates itself as learners interact with the system; a tutor model is the strategic part of an ITS which determines which instructional methods should be used; and finally a user interface model decides on the appearance and the type of interaction mode which ITS has with learners.

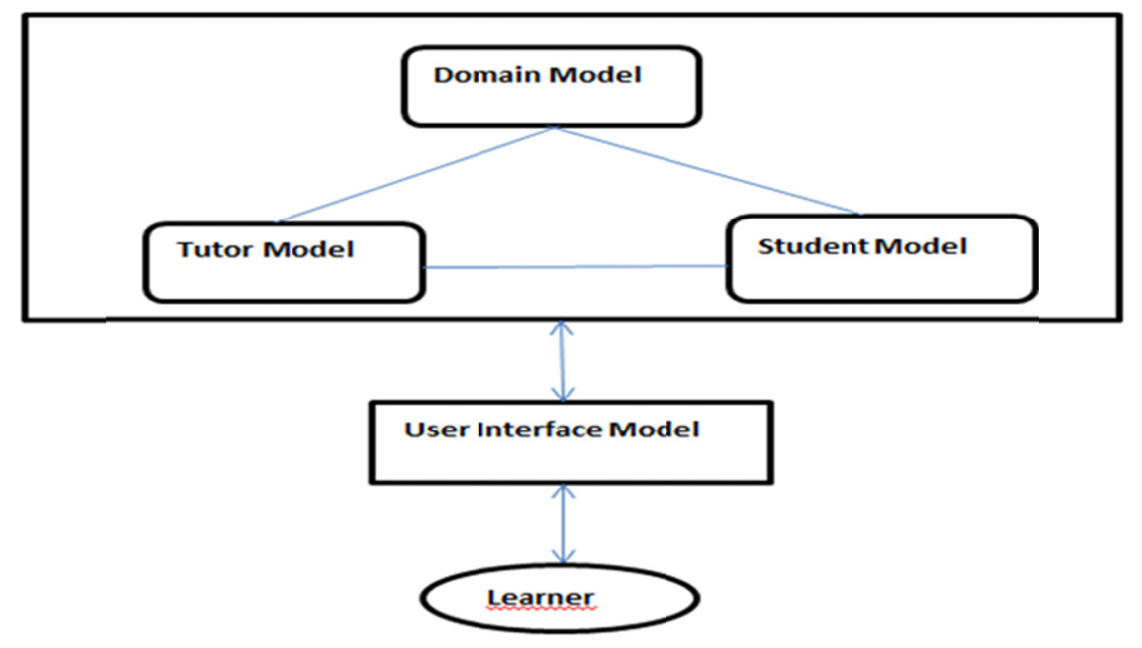

Figure 1. Architecture of Intelligent Tutoring Systems. Adapted from Mathews (1993)

\subsection{Adaptive Hypermedia}

Adaptive Hypermedia (AH) technologies are the new generation of hypermedia technologies which combine the research in Hypermedia and User Modeling (UM) to adapt the hyperlinks and the content of the hypertext to the needs, goals, interests, and facilities of the users (Brusilovsky, 1996, 1998, 2001, 2003). The technology of hypermedia emerged by the emergence of the World Wide Web (WWW) and found applications in different domains such as education, commerce, business, information, advertisement, etc. At the beginning, the technology was static and inflexible to the needs of individual users. For examples, if the users of WWW opened a particular information page, the same set of hyperlinks would be suggested to all of them.

This trend changed; however, as the advances in UM entered this field and the system could 
keep a profile of each user which held different types of information about him or her. This profile or model of user enabled the system to adapt its hyperlinks and content to the needs of individual learners, increasing the efficiency of these systems. Adaptive hypermedia technologies have been used for educational purposes as well, under the title of Adaptive Educational Hypermedia or simply Adaptive Learning Systems. In fact, these technologies were very influential in the emergence of ALSs, which are the topic of the next section.

\subsection{Adaptive Learning Systems}

ALSs are a new generation of ITSs which use technology, learning theories, and psychological insights to create instructional systems which adapt their appearance, content, content delivery, and assessment to the knowledge level and personal traits of the learners.

Researchers have described ALSs in different ways. According to Sonwalkar (2005), ALSs are systems which are designed to adapt to learners' preferences and customize learning content according to these preferences. Weibelzahl et al. (2008) define ALSs as tools to individualize the learning process by tailoring the instruction to learners' preferences, needs, knowledge, and goals. For Chieu (2005), "adaptability is the ability of a learning system to provide each learner with appropriate learning conditions to facilitate his or her own process of knowledge construction and transformation" (p. 70). The common theme to be extracted from the above and similar definitions of ALSs is a consideration of learners which makes a system capable of adjusting its content and instructional methods to the needs, proficiency level, learning styles, goals, and preferences of the learners; similar to what a human tutor does.

ALSs were partly motivated by the more general field of Web-based Education (WBE) that emerged by the spread of internet. Internet revolutionized information by making it accessible to anyone who had any sort of internet access from any kind of device regardless of time and place. This provided a great opportunity for the field of education, since it made information available to many people who did not have the time and resources of attending real classes, being tutored by real instructors, or having access to real instructional materials. Consequently, it led to the emergence and spread of WBE which made this access possible. However, an outstanding shortcoming of pure WBE technologies was their lack of adaptability and intelligence, which made them not efficient enough because such systems are supposed to be used by thousands of learners worldwide, and without some degrees of personalization and adaptiveness, they cannot achieve much success. ALSs emerged to compensate for the shortcomings of the previous systems. Though their hybrid architecture is rather new, they are not totally new in nature and have roots in two more traditional fields, Intelligent Tutoring Systems and Adaptive Hypermedia (Brusilovsky, 1999).

Sonwalkar ([INT 4], [INT 7], [INT 8], [INT 9], [INT 25]) in a series of video tutorials introduces four components which are critical to any adaptive learning system; namely educational framework, assessment, feedback, and revision.

Any adaptive tutoring system to be successful needs a sound pedagogical framework that appropriately leads the learners to achieve their learning goals. By an educational framework 
is meant the theoretical backbone of the system that justifies its choice of activities and decisions. Three dominant models of human learning; that is behaviorism, cognitivism, and constructivism, have been utilized and tested by different researchers to determine the best model of learning, and while most of the recent research sees constructivism as the best theory of learning to be used for adaptive learning systems (Matrins et al., 2008), others prefer a blended approach incorporating all three models (Sonwalkar, 2005).

Other than human learning models, an adaptive educational framework should be based on one or more categorizations of human learning styles to tailor its content delivery to the needs and preferences of individual learners. Sonwalkar ([INT 25]) proposes six learning styles that can be used in an adaptive learning system and are based on a blended-view model of human learning: apprenticeship, incidental, inductive, deductive, and discovery models of learning.

Assessment in an adaptive learning system happens when the performance of each learner in any of the learning styles used by the system is measured and the result is stored in the student model of the learner. For example, a concept is presented to a learner via apprenticeship and if the result is not satisfactory the same concept is presented via another learning style, such as inductive. This procedure continues and each time the result is stored in the learners' profile and statistically analyzed. After several interactions with the system and statistical assessments, the system can get a picture of each learner and figures out which learning style best matches his or her personal traits.

A third component of adaptive learning systems is immediate and adapted feedback given to learners while they interact with the system in order to inform them about their errors and improve their future performance. Here the learners can also get notified about their best personal learning strategies and styles, because according to Sonwalkar ([INT 4]), learners are not usually aware of the learning styles via which they learn best, and such information provided for them by the system through statistical analysis can be a valuable mean for their future learning.

The last component of a good adaptive learning system is to use the information which it acquires about learners' learning styles and knowledge to improve and tailor its performance. This component is very important, because the most sophisticated pedagogical framework and statistical procedures would be of little use if they are not used to revise the system behavior and adapt it to the needs and goals of the learners.

ALSs are the hot topic in technology-based instructional systems; therefore, the following section is devoted to a discussion of some of the most popular adaptive learning platforms currently in use. During the past decades, a large number of intelligent and adaptive educational systems have been developed, with fewer numbers being actually released and utilized by a large number of learners. Many of the systems ended up as laboratory experimental platforms or used in restricted domains. Information about those restrictedly used systems is not well available, so the following parts describe five instructional platforms which claim to be adaptive and capable of personalizing education to learners ([INT 20]). All of the selected systems are already being released and used by large numbers of learners and institutions. The information about them is taken from the websites of the companies and the 
forums where the actual users of these systems post their opinions and experiences of working with them. The descriptions; however, do not have a technical nature and depending on the amount of information available, may differ in depth and length.

\section{State of the Art in Intelligent and Adaptive Tutoring Systems}

\subsection{Knewton Adaptive Learning Platform}

Knewton is an adaptive-learning provider company founded in 2008 in New York City. Knewton calls itself the World Leading Adaptive Learning Provider and claims that its adaptive learning platform is capable of adapting content and instructional procedure to the knowledge level, goals, and characteristics of its users. Knewton is an LMS which is already used by a number of outstanding companies, publishers, and educational institutes such as Pearson, Gutenberg, Microsoft, Cambridge University Press, Wiley, Macmillan, etc., which use it in order to boost their user' learning and performance. Besides, Knewton designers and developers claim that their platform can be used to teach almost any subject, from mathematics to geography and history. Knewton obtains its adaptability power through detecting the users' performance. It can detect and record different types of users' actions such as whether they answer a question right or wrong, the amount of time they spend on a task, the learning style which best helps each user to learn a concept, or even the mouse movements when they are hesitating about selecting from a number of options. All these actions are recorded in each user's profile or student model and are compared with what the user has done before, and how other users have performed in similar situations. The results of such comparisons and analyses are used for adaptation, which occurs in different forms. For example, when a user stumbles with a particular task, the system adapts not only the level of difficulty of the next task, but also the type of it. In other words, if the system detects that a learner has problem with textual tasks, it may present the next task in visual or auditory format ([INT 10]).

Knewton is already a successful system with outstanding partners investing in it and using its platform for educational purposes. However, it is not void of criticisms. Tom Simonite (2012, [INT 11]) points to the fact that Knewton's claim about boosting students' learning and performance has not been tested in truly experimental situations yet. Though, in the website of this company there are some statistics about how users' performance has improved before and after using Knewton, Simonite (2012) mentions that in none of the experiments done so far there has been a control group to confirm the result. In other words, the groups using Knewton have never been compared with groups not using Knewton at the same time, but have been compared with previous groups of learners. The improvement in performance; therefore, could have been due to other factors such as ease of the materials, ease of assessment, or the effect of technology itself, not the Knewton adaptive learning platform. Another concern mentioned by Richard Clark (as mentioned in Simonite, 2012 [INT 11]) is that the company procedure to gather and analyze users' data is not evident and the company is not willing to shed light on that. 
Despite these criticisms and concerns, one cannot deny that Knewton is among the very few adaptive learning platforms which have found its way out of laboratories into the real world; and since it is yet in a very young age, any concrete conclusion about its efficiency or inefficiency is not possible. However, further experiments in truly controlled situation are critical in order to see whether it is more a mean of success or a tool of achieving money for the company investors.

\subsection{McGraw-Hill Education ALEKS}

McGraw-Hill Education is a digital learning company located in New York City with numerous partners around the globe which benefit from its educational content, software, adaptive learning services, and platforms. ALEKS is an adaptive learning platform to teach mathematics, science, and business courses, developed from research at New York University and University of California, and recently released by McGraw-Hill Education. Developers of ALEKS claim that it is unlike any other adaptive learning platform released so far because it is developed by a multi-disciplinary team of computer scientists, mathematicians, and cognitive psychologists, and at its heart lays an artificial intelligence engine.

ALEKS power comes from its ability to use AI to analyze the knowledge state of each learner in order to determine what she already does or does not know. To start with ALEKS, each learner takes part in an assessment test consisting of 20-30 questions. The test is not fixed, rather adapted to each learner; that is, each question in the test depends on how the student responds to the previous questions. ALEKS avoids multiple choice questions and after the initial assessment, it is capable of exactly determining the knowledge level of the learner with regard to any particular course in focus. After the initial assessments, ALEKS constantly observes the learners as they interact with the system and uses its data to present the learners only with the tasks which they are ready for. Also, it assesses the learners periodically to ensure that the learnt concepts can be retained ([INT 12]).

Similar to Knewton, ALEKS has its own problems and critics. A problem that many users of ALEKS have pointed to in a forum is that it works fantastically when it is used to review the already learned materials or to fill the gaps, but it is not that efficient as a stand-alone instructor. In other words, as users confront a concept that they have not learnt before, ALEKS provides them with some explanations and examples, but there are no more alternatives if the users do not understand those explanations and examples. On the other hand, an advantage of ALEKS mentioned by many users is the generation of statistics and graphs which show the users' progress and mastery or non-mastery of different concepts. Many ALEKS users believe that it is a good tool for parents and teachers, as well as the learners, to find out how far they have gone with the topics ([INT 13]).

\subsection{Smart Sparrow}

Smart Sparrow is an adaptive intelligent educational platform founded by a group of intelligent tutoring systems and educational data mining researchers at the University of New South Wales in Australia, to teach medicine and science courses. Smart Sparrow is designed to provide adaptive and interactive learning experiences for its users by giving them 
immediate feedback as they interact with the materials.

Its developers claim that Smart Sparrow has three levels of adaptiveness; namely, instant adaptive feedback, adaptive pathways, and the possibility for instructors to adapt their teaching. In the first level of adaptiveness, the system offers the students intelligent and immediate feedback as they face a problem in doing a task. The second level of adaptiveness caters for individual learners' differences. It can detect each learner current level of knowledge, misconceptions, and learning rate, and adapt the level of difficulty and the delivery of the material to them. In the third level of adaptiveness, the system takes the teachers and instructors into consideration. Smart Sparrow is equipped with powerful authoring tools and facilities which enable the teachers to add their content, feedback, videos, simulations, etc., into the system to provide more individualized and interactive learning environments for their students ([INT 14]).

Smart Sparrow is currently being used in many of educational institutes in Australia, with the perspective of reaching the global markets, and has attracted good attention and investment. However, in comparison to some other adaptive learning systems, this system is not that sophisticated in adapting itself to learners' differences as it is to help the instructors create interactive courses. Its adaptiveness power lays mostly in its ability to give immediate feedback; and as the system founder has mentioned, the platform is no more than a rule-based system and void of true intelligence.

\subsection{Desire2Learn \& Knowillage Leap}

Desire2Learn Company which is the creator of an integrated learning platform made a partnership with Knowillage Company, the creator of an adaptive learning platform, in September 2013 to form the Desire2Learn \& Knowillage Leap adaptive learning platform. Originally founded in Canada, Desire2Learn company has now over 750 clients and ten million users in higher education, healthcare, and K-12 in Canada, the United States, Europe, Australia, Brazil, and Singapore ([INT 15], [INT 16], [INT 17]). Creators of Desire2Learn \& Knowillage Leap platform believe that the advances in technology have not been well utilized in the domain of education and most of what already exists is restricted to learning management systems which automate simple tasks or provide simple authoring tools, without engaging the learners and adapting the course to their needs. Their adaptive learning platform provides a rich inventory of tools for instructors to create engaging courses and materials using the latest technology. Also, this platform is equipped with a discussion forum where the students can be in contact with instructors or other students. To adapt content to each learner, this platform utilizes pre and post assessment and uses the results to determine the learning path of each learner.

A typical interaction with Desire2Learn \& Knowillage Leap platform is as follows: the instructor adds a particular learning path to a course which already exists. The instructor has several resources to add to this new path such as external links, pre and post assessment tests, videos, audios; to mention a few. After the learning path is added, the students can access that. They usually start with a pre-test which becomes a basis to adapt the learning path to their knowledge level. Then, they enter the learning path and go through different tasks which are 
adjusted and adapted to them. At each level of learning, they have access to practice exercises, and this trend continues until they master the particular topic. The mastery is proved if they can pass the post-test, and afterward they can go to the next level of the course ([INT 15], [INT 16], [INT 17]).

Overall, data shows that up to this point the Desire2Learn \& Knowillage Leap platform does not benefit from any truly intelligent and adaptive engine. One can consider it more as a sophisticated learning management system which offers more features to instructors and learners, than an intelligent and adaptive learning system. Additionally, many users of it have mentioned that they have encountered numerous technical problems working with it, due to its poor maintenance and bugs. The combination of Desire2 Learn company with Knowillage Leap Company is; nevertheless, new, and in future one may observe the resolution of the current problems and introduction of more intelligent and adaptive features into this platform.

\subsection{DreamBox Learning}

DreamBox Learning is an adaptive learning platform to teach mathematics to elementary students, founded in 2006 in Washington. It uses animations and games to teach mathematics and is based on the principle of "learning by doing". Students working with DreamBox go through missions, challenges, awards, and punishments to acquire mathematical concepts, and the system keeps them engaged and interested while learning. DreamBox Learning claims to be adaptive and intelligent and is equipped with millions of learning pathways to personalize learning for each learner. DreamBox Learning is based on three principles of rigorous elementary mathematics, motivation learning environment, and intelligent adaptive learning. Based on the first principles, DreamBox Learning attempts to keep the quality and difficulty level of its math content in line with national and international standards. The second principle provokes DreamBox Learning platform to create a learning environment which is engaging and motivating, as well as informative and instructive. The system has achieved this goal through a game-like environment where the learners learn while playing and having fun. The third principle is where the system intelligence and adaptiveness comes from. Students are constantly assessed while working with the system and the results of these assessments are used to adapt the level of difficulty and the pace of instruction to each learner. DreamBox Learning has millions of learning paths, and at each stage, the learners are guided to one of these paths based on the system assessment of them and based on their unique needs. The system is also cautious that each student is moving under the proximal zone of development, introducing at each stage only those tasks for which the learner is ready ([INT 18]).

DreamBox Learning has been successful so far and most of the reviews about it reflect the positive attitudes of the users. One flaw which; however, some have mentioned is that it cannot be used as the only source of instruction because it does not provide additional alternatives for the users if they cannot grasp a concept. They believe that DreamBox is a marvelous tool if it is used in a blended learning environment where the learners have access to other knowledgeable bodies when they encounter problems. 


\section{Conclusion}

The advances in computer technology and the spread of internet have affected every single aspect of human life. People have an unprecedented access to information from any place and time which is unlike any time before. Education, as an important part of human societies and individuals' lives, is also affected by these technologies, because from the early days of their emergence, educators and scientists began to realize the potentials which they have for education. Since then, technology has been integrated into education in different forms and levels, being now an inseparable part of that.

This article attempted to outline and review one class of educational technology which is the field of intelligent technology-based instruction. The field of intelligent technology-based instruction was further divided into two sub-fields of intelligent tutoring systems (ITSs) and adaptive learning systems (ALSs). Besides, a number of adaptive learning platforms which are currently used by a large of number of actual users were introduced and it was attempted to demonstrate to what extent they adhere to the theoretical principles behind adaptive intelligent learning systems. Also, the strong and weak points of these platforms and how future systems can improve them were discussed.

A comparison of the adaptive learning platforms introduced demonstrates that almost all of them adhere to the four-component framework introduced by Sonwalkar ([INT 4], [INT 7], [INT 8], [INT 9], [INT 25]).

A closer look at the Knewton adaptive learning platform shows that the system is mostly based on Cognitivism theory of learning with its emphasis on tailoring education to learners' goals, needs, learning styles, and providing them with appropriate feedback. Also, adaptiveness occurs to tap not only the learners' differences in knowledge level, but also their differences in desired learning styles. The principles of adapting instruction to learners and the benefits of one-to-one instruction are; also, taken into account. This adaptive learning platform assesses the learners before starting the main courses and during the courses, and revises its behavior based on the assessment results. In addition, Knewton provides feedbacks for learners as they work with the system, as well as giving them recommendations about what to do next to improve their performance.

ALEKS adaptive platform; similarly, benefits from a pedagogical framework which is mostly in line with Cognitivism theory of learning. It cares about the learners by adapting its instruction to their goals, knowledge level, and providing them with feedbacks. However; in contrast to Knewton, the adaptation is not based on the learning styles of individuals, but only on their knowledge level. Additionally, the system regularly assesses the learners both before taking the course and as they interact with the system, and uses this assessment to adapt its instruction. Feedback is provided after each course in the form of graphs and charts which show how much the learner has progressed and in which areas he or she needs more practice.

The next platform, discussed above, is the Smart Sparrow adaptive platform which is developed in Australia. Smart Sparrow is based on the principle of learning by doing which makes it closer to the Constructivism theory of learning. This platform is used mainly to 
teach at medicine and engineering schools and helps learners by taking them into real-world-like simulation environments where they can practice what they may encounter in the real world later. This platform uses no explicit assessment but assesses the learners regularly as they interact with the system and adapts its instruction based on the results. Adaptation; however, is only based on what the learners know or do not know, and not on other learners' characteristics such as learning styles. Feedback is given instantly as the learners face a problem during the interaction. Smart Sparrow; however, as the founders of it explain, is mostly a rule-based system and its intelligence comes from the pre-defined rules which are given to it.

Desire2Learn \& Knowillage Leap has recently developed an adaptive learning platform which is adaptive to learners' differences in their knowledge level. The system uses pre-tests before any course to measure each learner knowledge level and adapts the course to the results obtained. Also, practice tests are available to assess learners' progress during the courses, and post-tests which are used as an indicator of whether a learner should go to the next level or not. Tests are mainly in multiple-choice format and are a criterion for the system to adjust its content. Additionally, feedback is presented as graphs which show progress and weakness in any area. This learning platform is equipped with a discussion forum where the users can be in touch and interchange their opinions.

DreamBox Learning is the last adaptive learning platform discussed above which is developed to teach mathematics to elementary students. This system uses games and animation to teach mathematics and is based on the principle of learning by doing. Learners go through a series of missions and adventures and at the same time learn mathematical concepts. The system assesses them regularly as they interact and adjust its instruction based on the results, but no formal test is given. Feedback is not given to the learners themselves, but is sent for the parents in the form of progress reports.

Comparison of the five popular adaptive learning platforms confirms that the four components of good adaptive learning systems is considered in the design of these platforms, though a consideration of learning styles is only included is one of them. In other words, most of these systems use some sort of direct or indirect assessment to adapt their behavior, but the only learner characteristic which they take into account is knowledge, which is definitely not enough, because as said elsewhere a good human instructor is also capable of recognizing learners' differences in learner styles and adjust instruction to them. This is a deficiency which should definitely be considered in future adaptive systems if the goal is to provide the benefits of one-to-one instruction for any one at any place. Another problem which many users of these platforms mention is that they cannot efficiently be used as standalone instructors and almost always there is a need for a third party instructor to help learners grasp the concepts fully. This is also in contrast to the overall goal of adaptive learning system theory, because one of the main motivations behind these systems is to be used instead of human instructors where and when the learners do not have access to them.

Adaptive learning systems are at present in a stage which definitely needs to be upgraded. The process of creating effective learning systems is one that undoubtedly requires the 
cooperation of many different disciplines such as education, psychology, computer science, AI, etc. Many of the current systems are powerful in some aspects of their design, but still poor in others, and this is due to the lack of proper cooperation among those disciplines. In addition, learners are a basic factor which should be integrated at every single aspect of adaptive learning systems' design. It is essential to recognize that learners are multi-dimensional beings and categorizing them solely based on their knowledge level is definitely false. There are many personal traits which should be integrated in adaptive learning systems to enhance their effectiveness.

\section{References}

Bloom, Benjamin S. (1984). The 2-Sigma Problem: The Search for Methods of Group Instruction as Effective as One-to-One Tutoring. Educational Researcher, 13(6), 4-16. http://dx.doi.org/10.3102/0013189X013006004

Brusilovsky, Peter. (1996). Methods and Techniques of Adaptive Hypermedia. Journal of User Modeling and User-Adapted Interaction, 6(2-3), 87-129. http://dx.doi.org/10.1007/BF00143964

Brusilovsky, Peter. (1998). Adaptive Educational Systems on the World-Wide-Web: A Review of Available Technologies. Paper Presented at the 4th International Conference on Intelligent tutoring Systems. 1998. San Antonio. Retrieved from http://www1.cs.columbia.edu/ locasto/projects/web-education/papers/brusilovsky98ada ptive.pdf Last Access: 28 May 2014

Brusilovsky, Peter. (1999). Adaptive and Intelligent Technologies for Web-based Education. In C. Rollinger and C. Peylo (eds.), Special Issue on Intelligent Systems and Teleteaching, Künstliche Intelligenz, (4), 19-25.

Brusilovsky, Peter. (2001). Adaptive Hypermedia. User Modeling and User Adapted Interaction. Ten Year Anniversary Issue (Alfred Kobsa, ed.), 11(1/2), 87-110. http://dx.doi.org/10.1023/A:1011143116306

Brusilovsky, Peter. (2003). Developing Adaptive Educational Hypermedia Systems: From Design Models to Authoring Tools. In Thomas Murray, Stephen Blessing, S. Ainsworth. (eds.), Authoring Tools for Advanced Technology Learning Environments: Toward Cost-effective Adaptive, Interactive, and Intelligent Educational Software. Dordrecht, Kluwer. 2003. pp. 377-409.

Chieu, Vu M. (2007). Constructivist Learning: An Operational Approach for Designing Adaptive Learning Environments Supporting Cognitive Flexibility. Educational Technology \& Society, 10(3), 32-46.

Elsom-Cook, Mark. (1987). Intelligent Computer-aided Instruction Research at the Open University. Technical Report No: 63. Computer-Assisted Learning Research Group. The Open University: Milton Keynes.

Martins, A. C., Faria, L., Carvalho, C. V., \& Carrapatoso, E. (2008). User Modelling in Adaptive Hypermedia Educational Systems. Educational Technology \& Society, 11(1), 194-207. 
Mathews, Clive. (1993). Grammar Frameworks in Intelligent CALL. Journal of Computer Assisted Language Instruction Consortium, 11(1), 5-27.

Nwana, Hyacinth. S. (1990). Intelligent tutoring systems: An overview. Journal of Artificial Intelligence Review, 4, 251-277. http://dx.doi.org/10.1007/BF00168958

Psotka, J., Massey, L. D., \& Mutter, S. A. (eds.). (1988). Intelligent Tutoring Systems: Lessons Learned. Hillsdale, NJ: Lawrence Erlbaum Associates, Inc.

Self, John A. (1974). Student Models in Computer-aided Instruction. International Journal of Man-Machine Studies, 6, 261-276. http://dx.doi.org/10.1016/S0020-7373(74)80005-2

Sleeman, D. H., \& Brown, J. S. (eds.). (1982). Intelligent Tutoring Systems. New York: Academic Press.

Sonwalkar, Nishikant. (2005). Adaptive Learning Technologies: From One-size-fits-all to Individualization. Educause Center for Applied Research, 7, 1-11.

Urban-Lurain, Mark. (1996). Intelligent Tutoring Systems: A Historic Review in the Context of the Development of Artificial Intelligence and Educational Psychology. Retrieved 27 May 2014 from http://www.cse.msu.edu/rgroups/cse101/ITS/its.htm

Weibelzahl, S., De Bra, P., Paramythis, A., Ertmer, P., \& Desjardins, F. (2008). Adaptive or Collaborative Learning? Proceedings of World Conference on Educational Multimedia, Hypermedia and Telecommunications, 5474-5477.

\section{Internet Sources}

[INT 1] http://its2014.its-conferences.com/, 21 May 2014.

[INT 2] http://en.wikipedia.org/wiki/B__F._Skinner, 21 May 2014.

[INT 3] http://www.personalityresearch.org/papers/naik.html, 21 May 2014.

[INT 4] https://www.youtube.com/watch?v=lIVaY3Qci6M, 21 May 2014.

[INT 5] http://en.wikipedia.org/wiki/Learning_styles, 21 May 2014.

[INT 6] https://www.youtube.com/watch?v=zxUW_z9iA2Q, 21 May 2014.

[INT 7] https://www.youtube.com/watch?v=fnWMXQGDSTk, 21 May 2014.

[INT 8] https://www.youtube.com/watch?v=BUVDubKL1Yw, 21 May 2014.

[INT 9] https://www.youtube.com/watch?v=YiMWhacSwrc, 21 May 2014.

[INT 10] http://www.knewton.com/, 21 May 2014.

[INT 11] http://www.technologyreview.com/news/506366/

questions-surround- software-that-adapts-to-students/, 29 May 2014.

[INT 12] http://www.aleks.com/, 29 May 2014.

[INT 13] http://forums.welltrainedmind.com, 21 May 2014.

[INT 14] https://www.smartsparrow.com/, 29 May 2014. 
[INT 15] http://www.knowillage.com/leap_d21.html, 21 May 2014.

[INT 16] http://www.Desire2Learn.com, 21 May 2014.

[INT 17] http://www.desire2learn.com/pressrelease/desire2learnacquires-knowillage-systems-inc /, 29 May 2014

[INT 18] http://www.dreambox.com/, 21 May 2014.

[INT 19] http://www.cse.msu.edu/rgroups/cse101/ITS/its.htm, 21 May 2014.

[INT 20] https://www.educationdive.com/news/adaptive-learningthe-best-approaches-weve-seen-so-far/187875/, 21 May 2014.

[INT 21] http://www1.cs.columbia.edu/ locasto/projects/web-education /papers/brusilovsky98adaptive.pdf, 21 May 2014.

[INT 22] https://www.academia.edu/4313503/Tutoring_and_Expert_Module _of_Intelligent_Tutoring_Systems, 21 May 2014.

[INT 23] http://www.cs.utexas.edu/users/ml/papers/assert-dissertation-94.pdf, 21 May 2014.

[INT 24] http://www.cse.lehigh.edu/ glennb/designfirst/PARVEZ_ITS2008.pdf , 21 May 2014.

[INT 25] https://www.youtube.com/watch?v=cGwibPdHS8Y, 21 May 2014.

\section{Copyright Disclaimer}

Copyright for this article is retained by the author(s), with first publication rights granted to the journal.

This is an open-access article distributed under the terms and conditions of the Creative Commons Attribution license (http://creativecommons.org/licenses/by/3.0/). 